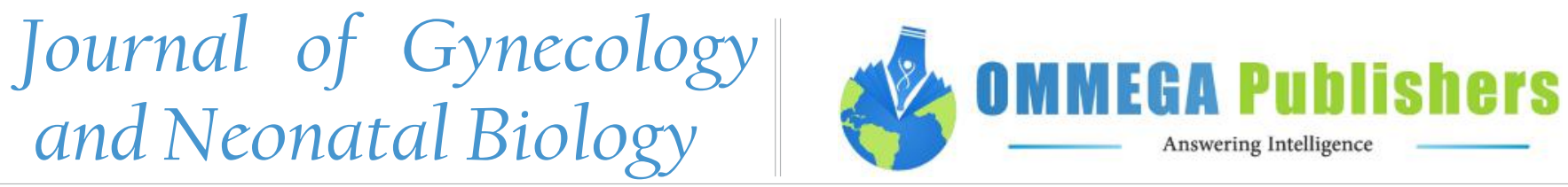

Editorial

\title{
Premature Ovarian Failure of Autoimmune Causes
}

\author{
Mara Carsote $^{1 *}$, Ana Valea ${ }^{2}$ \\ ${ }^{1}$ Department of Endocrinology, Carol Davila University of Medicine and Pharmacy \& C.I.Parhon National Institute of Endocrinology, Bucharest, \\ Romania \\ ${ }^{2}$ Iuliu Hatieganu University of Medicine and Pharmacy \& Clinical County Hospital, Cluj-Napoca, Romania
}

*Corresponding Author: Carsote, M. Department of Endocrinology, Carol Davila University of Medicine and Pharmacy \& C.I.Parhon National Institute of Endocrinology, Bucharest, Romania. Tel: +40213172041; Fax: +40213170607; E-mail: carsote_m@hotmail.com

Citation: Carsote, M. et al. Premature ovarian failure of autoimmune causes (2015) J Gynecol Neonatal Biol 1(1): 14-15.

Received date: June 16, 2015

Accepted date: June 18, 2015
Published date: June22, 2015

\section{Editorial}

Complex and dynamic interrelationships are seen between gonad hormones and hormones that control reproductive functions and immune system. The best example for this is the premature ovarian failure (POF) of autoimmune causes. Generally POF (or premature ovarian insufficiency) represents the clinical end stage of ovarian function in women less than 40 years old ${ }^{[1-4]}$. The estrogens are low and the Follicle Stimulant Hormone (FSH) is high (above $40 \mathrm{mIU} / \mathrm{mL}$ ) while the clinical main feature is the absence of menses for several months and hypoestrogenic state associated symptoms ${ }^{[2-4]}$. The decreased number of ovarian follicles or follicular dysfunction are the pathogenic backgrounds for the condition ${ }^{[1-4]}$. POF affects $1-3 \%$ of adult women with an increasing frequency during the last decades in same populations or subjects with certain previous medical conditions (generaly is considered as having a frequency 1 of 100 at $<40$ years, and 1 of $1000<30$ years $)^{[5,6]}$.

The POF aetiology comprises 6 classes of causes: iatrogenic (increasing frequency during the last years), idiopathic (currently considered as most frequent), genetic, environmental, infections and autoimmune $(4-30 \% \text { of all })^{[1-4,7,8]}$. The exact mechanisms of autoimmune POF remain obscure: probably the genetic and environmental factors initiate the immune response $^{[2,7-9]}$. It Involves: major histocompatibility complex antigen, cytokines, cell-mediated immunity, antibody-mediated immunity ${ }^{[2,7-9]}$. Based on autoimmune context there are three types of autoimmune POF: adrenal autoimmune POF, ovarian and isolated; the combination of auto antibodies+autoimmune diseases+ovarian histology making the disease 1.possible, 2.probable, or 3.confirmed ${ }^{[9]}$. Autoimmune oophoritis includes mononuclear inflammatory cell infiltrate in the theca cells of growing follicles, early stage follicles without lymphocytic infiltration, final infiltrate with plasma, B and T-cells, auto antibodies to ovarian antigens (as markers of ovarian autoimmunity); potential others organ specific autoimmunity ${ }^{[7,8,10]}$. Anti-ovarian antibodies offer no prognostic of POF; are provided by simple blood samples as ELISA; their detection varies from $20-70 \%$ due to cyclic variations in antigenic proteins; their current practice has limited use (there is no valid marker); and numerous triggers have been described as viruses (mumps and rubella, bacteria, and self-ovarian antigens $)^{[2,7-9,11]}$. Antigenic targets for antibody in autoimmune POF are steroid producing cells (as seen in adrenal autoimmune disease); 3 beta-hydroxysteroid dehydrogenase; gonadotropin receptor blocking antibodies (very rare), other ovarian antigens as zona pellucida, corpus luteum ${ }^{[9-11]}$. Murine models reproduced the autoimmune ovarian condition for instance by exposure to ZP3 peptide, and later a reversible disease was found by orally induced tolerance to ZP3 peptides which suppresses the experimental gonad disease ${ }^{[12,13]}$. The reversibility in humans is rarely seen only in some cases with autoimmune associated conditions that are controlled. The autoimmune panel of medical co-morbidities is complex: endocrine and non-endocrine as chronic candidiasis, vitiligo, alopecia areata, autoimmune haemolytic anaemia, systemic lupus erythematosus, Sjogren syndrome, miastenia gravis, Crohn's disease, primitive billiary cirrhosis, autoimmune hepatitis, etc ${ }^{[7,8]}$. The endocrine combinations are type 1 (or autoimmune polyendocrinopahy - candidiasis - ectodermal dystrophy) and type 2 autoimmune polyglandular syndrome ${ }^{[8,14]}$. Addison's disease associated POF is presented in $10 \%$ of cases and POF correlates autoimmune adrenalitis in 2-20\% of situations because both ovaries and adrenals expose common antigens with steroid-producing cell antibodies and steroidogenic enzymes antibodies (as 17 $\alpha$-hydroxylase autoantibodies or P450 side-chain cleavage enzyme autoantibodies $)^{[9,15]}$. Autoimmune adrenal oophoritis involves a selective destruction of the theca cells with a specific preservation of granulosa cells (that produce low amount of estradiol) $)^{[9,15,16]}$. The markers of ovarian reserve are high serum inhibin $\mathrm{B}$ (compared to healthy fertile women and other forms of immune oophoritis) and normal serum AMH (anti-mullerian hormone) in $2 / 3$ of cases indicating a preserved pool of functioning follicles ${ }^{[9,15,16]}$. The thyroid auto-

Copy rights: (C2015 Carsote, M. This is an Open access article distributed under the terms of Creative Commons Attribution 4.0 International License. 
immunity associated POF is the easiest to assess by testing the thyroid antibodies, every new case of POF should be checked by TSH, free T4, anti-thyroid-peroxidase antibodies, anti-thyroglobuline antibodies ${ }^{[17]}$. Thyroid autoimmunity is rarely seen in type 1 polyendocrine autoimmune syndrome and frequent found in type 2 (at risk with HLA DR3 and DR4 and protective by HLA DR2) $)^{[17]}$.

Current clinical practice in autoimmune POF comprises the following key points: the gold standard is ovarian biopsy but is not recommended; commercially available antiovarian antibodies have a poor predictive value; there is no validated serum marker; it is better to search for the general definition criteria and associated potential autoimmune context ${ }^{[9,15]}$. Specific therapy of autoimmune POF apart from general multidisciplinary approach of POF with other causes theoretical involves the need to restore ovarian function after immune suppression but no corticosteroids, neither immune-modulator therapy are currently useful only some immunosuppressive therapies in selective population $^{[9,15]}$

Autoimmune POF is one of the most mysterious and yet challenging issues of reproductive and menopausal medicine. Idiopathic POF probably underlines others unknown autoimmune mechanisms and the future will highlight numerous aspects yet incompletely understood.

\section{Premature ovarian failure}

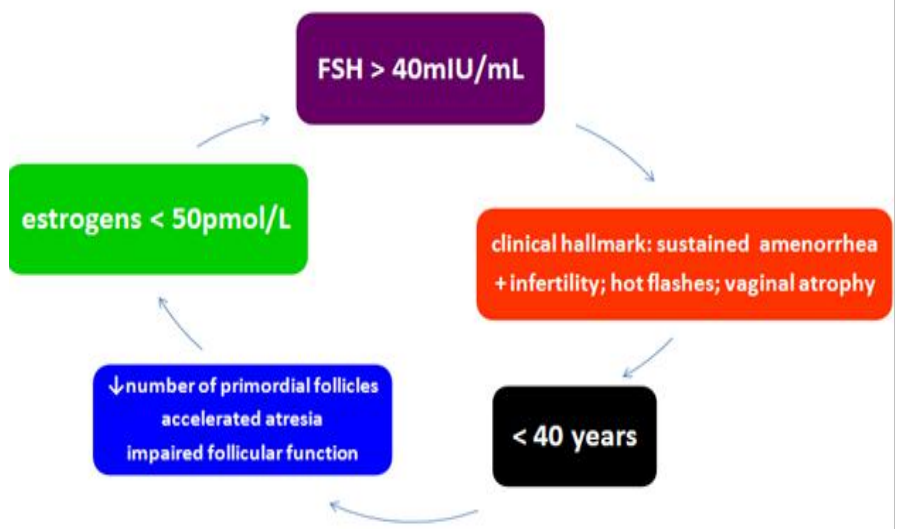

Figure 1: The elements in premature ovarian failure: secondary amenorrhea before the age of 40 years, potential climacteric symptoms as: hot flashes, etc; hormonal panel suggestive for hypergonadotropic hypogonadism and follicle dysfunction and decreased number related pathological disturbances

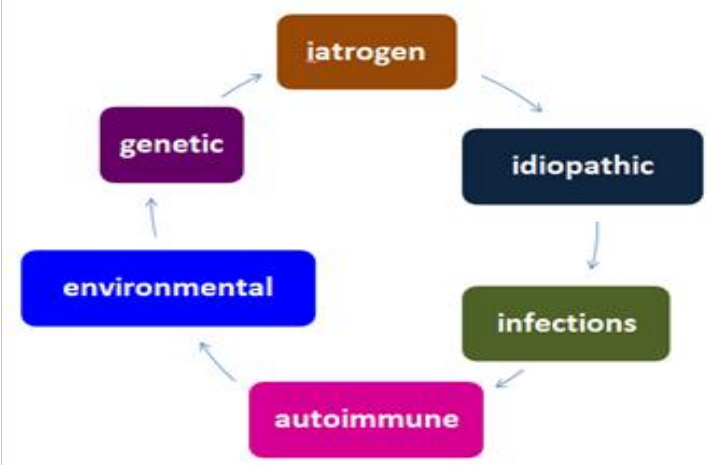

\section{References}

1. Slopień, R., Warenik-Szymankiewicz, A. Premature ovarian failure: diagnosis and treatment. (2014) Clin Exp Obstet Gynecol 41(6): 659661 .

2. Shamilova, N.N., Marchenko, L.A., Dolgushina, N.V., et al. The role of genetic and autoimmune factors in premature ovarian failure. (2013) J Assist Reprod Genet 30(5): 617-622.

3. Shelling, A.N. Premature ovarian failure. (2010) Reproduction 140(5): 633-641.

4. Maclaran, K., Panay, N. Current concepts in premature ovarian insufficiency. (2015) Womens Health (Lond Engl) 11(2): 169-182.

5. Coulam, C.B., Adamson, S.C., Annegers, J.F. Incidence of premature ovarian failure. (1986) Obstet Gynecol 67(4): 604-606.

6. Vujovic, S., Brincat, M., Erel, T., et al. EMAS position statement: Managing women with premature ovarian failure. (2010) Maturitas 67(1): 91-93.

7. Khole, V. Does ovarian autoimmunity play a role in the pathophysiology of premature ovarian insufficiency? (2010) J Midlife Health 1(1):9-13.

8. Silva, C.A., Yamakami, L.Y., Aikawa, N.E., et al. Autoimmune primary ovarian insufficiency. (2014) Autoimmun Rev 13(4-5): 427-430.

9. Ebrahimi, M., Akbari Asbagh, F. Pathogenesis and causes of premature ovarian failure: an update. (2011) Int J Fertil Steril 5(2): 54-65.

10. Bukulmez, O., Arici, A. Autoimmune premature ovarian failure. (2002) Immunol Allergy Clin N Am 22(3): 455-470.

11. Dragojević-Dikić, S., Marisavljević, D., Mitrović, A., et al. An immunological insight into premature ovarian failure (POF). (2010) Autoimmun Rev 9(11): 771-774.

12. Garza, K.M., Griggs, N.D., Tung, K.S. Neonatal injection of an ovarian peptide induces autoimmune ovarian disease in female mice: requirement of endogenous neonatal ovaries. (1997) Immunity 6(1): 89-96.

13. Fu, L., Feng, W., Li, S.R., et al. ZP3 peptides administered orally suppress murine experimental autoimmune ovarian disease. (2007) J Reprod Immunol 75(1): 40-47.

14. Arlt, W., Allolio, B. Adrenal insufficiency. (2003) Lancet 361(9372): 1881-1893.

15. Reato, G., Morlin, L., Chen, S., et al. Premature ovarian failure in patients with autoimmune Addison's disease: clinical, genetic, and immunological evaluation. (2011) J Clin Endocrinol Metab 96(8): E12551261.

16. La Marca, A., Brozzetti, A., Sighinolfi, G., et al. Primary ovarian insufficiency: autoimmune causes. (2010) Curr Opin Obstet Gynecol 22(4): 277-282.

17. Wémeau, J.L., Proust-Lemoine, E., Ryndak, A., et al. Thyroid autoimmunity and polyglandular endocrine syndromes. (2013) Hormones (Athens) 12(1): 39-45.

Figure 2 POF aetiology 